\title{
Study on the Swelling Behavior of Gamma Radiation Induced Acrylamide/Carboxymethyl Cellulose Blend Hydrogel in Urea Solution.
}

\author{
Salma Sultana $^{*}{ }^{\text {, Kumkum Habib }}{ }^{1,3}$, M. Rabiul Islam ${ }^{2}$, Nirmal Chandra Dafader ${ }^{1}$, M. Emdadul Haque ${ }^{1}$ and A. F. M. \\ Mustafizur Rahman*3 \\ ${ }^{I}$ Nuclear and Radiation Chemistry Division (NRCD), Institute of Nuclear Science and Technology(INST), Bangladesh Atomic \\ Energy Commission (BAEC), Dhaka, Bangladesh \\ ${ }^{2}$ Department of Chemistry, Jahangirnagar University, Savar, Dhaka, Bangladesh \\ ${ }^{3}$ Department of Applied Chemistry and Chemical Engineering, Dhaka University, Dhaka-1000. Bangladesh
}

(Received: 15 June 2015; Accepted: 29 May 2016)

\begin{abstract}
Swelling behaviors of crosslinked acrylamide (AAm)/carboxymethyl cellulose sodium salt (CMC) blend hydrogels synthesized using gammaradiation from Co-60 source in aqueous solutions of urea were investigated. The parameters like effect of radiation dose and concentration of $\mathrm{CMC}$ in $\mathrm{AAm} / \mathrm{CMC}$ blend were studied on swelling properties. Swelling experiments were performed in various concentrations of urea solution and distilled water at room temperature. The swelling properties of hydrogels increased with increased concentration of $\mathrm{CMC}$ but it decreased with increased radiation dose. Hydrogels showed enormous swelling in aqueous solution of urea. The swelling properties of $\mathrm{AAm} / \mathrm{CMC}$ hydrogel were lower in distilled water than that of in urea solution. The water absorption of AAm/CMC blend hydrogels increased from $\sim 4,800$ to $\sim 9,000 \%$ for the concentration range of $\mathrm{CMC}$ from 0 to $7 \%$ for 48 hours standing in distilled water at $25 \mathrm{kGy}$ radiation dose. It was also found that water absorption by AAm hydrogel with 7\% CMC decreased from $\sim 13,800$ to $~ 8,500 \%$ for the radiation range from 15 to $35 \mathrm{kGy}$ for 48 hours standing time in water. The equilibrium water content (EWC) of AAm/CMC blend hydrogel depended on radiation dose. EWC decreased from $\sim 0.9935$ to $\sim 0.9896$ with the variation of radiation dose from 15 to $35 \mathrm{kGy}$ in $0.01 \mathrm{M}$ urea solution at 48 hours.
\end{abstract}

Keywords: Acrylamide, Carboxymethyl cellulose sodium salt, Gamma-radiation, Hydrogel, Urea, Swelling behaviours

\section{Introduction}

Hydrogels are a class of hydrophilic polymeric materials that can absorb large amounts of water. These polymers do not dissolve in water; upon swelling, they increase in volume, but keep their shape without breaking up. Due to good swelling capacity, hydrogels can be easily used to remove reagent residues. On the other hand, the large water content makes hydrogels a special class of materials. Their network structure is the result not only of covalent bonding but also ionic, hydrogen or even vander Waals interactions. The unique physical properties of hydrogels such as high water affinity, high thermal and mechanical stability, biocompatibility, allies a variety of industrial and biomedical applications ${ }^{1-4}$.

Urea is one of the main toxic wastes in the dialysis solution from hemodialysis. The most effective way to remove urea from aqueous solutions is the utilization of immobilized urease as no efficient adsorbent is available for urea. On the other hand, urea has a great importance in biological systems ${ }^{5}$. Many researchers found that urea was not effective on certain solution properties, such as molecular association, viscometric characteristics, reduction of temperatures, polymer dimensions, and thermodynamic characteristics of water-soluble polymers compared with the other denaturing agents. However, studies are being widely carried out due to its biological importance ${ }^{6,7}$. Swelling ratio of polyvinyl pyrrolidone (PVP) hydrogel improves with addition of kappa-carrageenan (KC) due to the presence of$\mathrm{OSO}_{3}^{-}$group in $\mathrm{KC}$ molecule ${ }^{8}$. It is reported that swelling behaviour of PVP hydrogel increases with addition of acrylic acid ${ }^{9}$. It is also reported that the carboxymethyl cellulose (CMC) improves the swelling ratio of polyvinyl alcohol (PVA)/sago blend hydrogel due to the presence of the carboxyl group in the CMC molecule ${ }^{\mathbf{1 0}}$. In this study, the effect of radiation dose and concentrations of CMC in AAm/CMC blend were studied on swelling properties. Swelling experiments were performed in various concentrations of urea in solution and distilled water at room temperature.

\section{Experimental}

\section{Raw Materials}

Acrylamide was purchased from Aldrich Chemical and purity was 97\%. CMC was purchased from Sigma; Urea was purchased from Rankem, RFCL Ltd. Distilled water was used to prepare solution.

\section{Preparation of AAm/CMC blend hydrogel}

Homogeneous solutions containing $5 \%$ of acrylamide were prepared in distilled water with different concentration of CMC (3 and 7\%). Then the solutions were poured in several glass test tubes, sealed with polyethylene and finally irradiated with gamma-rays from Co-60 source by varying radiation dose of $10,15,20,25,30$ and $35 \mathrm{kGy}$ (dose rate $5 \mathrm{kGy} /$ hour $)$ at room temperature $\left(27^{\circ} \mathrm{C}\right)$. The irradiated samples were cut into small pieces, dried at room temperature and then in an oven at $50^{\circ} \mathrm{C}$ to constant weight for measurement of gel fraction and swelling properties.

\section{Determination of swelling ratio}

The swelling ratio of hydrogel was determined by gravimetric method. The dried gel to a constant weight was immersed in distilled water and other test solution until the maximum swelling was obtained at room temperature. Then

Author for correspondence. e-mail: sultanasalma71@yahoo.com; mustafizacce@du.ac.bd 
hydrogel sample was weighed after removing excess water on the surface of sample with a tissue paper. The swelling ratio was calculated as:

$$
\text { Swelling ratio }=\left[\left(\mathrm{W}_{2}-\mathrm{W}_{1}\right) / \mathrm{W}_{1}\right]
$$

Where $\mathrm{W}_{2}$ is the weight of swelled gel and $\mathrm{W}_{1}$ is the weight of dried gel.

\section{Determination of water absorption}

The gel sample was dried to a constant weight and was kept in distilled water at a room temperature and then the gel sample was periodically weighed after soaking the surface water by tissue paper. The water absorption was calculated as:

$$
\text { Water absorption }(\%)=\left[\left(\mathrm{W}_{\mathrm{t}}-\mathrm{W}_{1}\right) / \mathrm{W}_{1}\right] \times 100
$$

Where $\mathrm{W}_{\mathrm{t}}$ is the weight of swelled gel at time $t$ and $\mathrm{W}_{1}$ is the weight of dried gel.

\section{Results and Discussion}

Swelling Ratio of AAm and AAm/CMC blend hydrogels in urea solutions

Swelling is a result of balance between two forces. One is osmotic force and the other is dispersing force. Osmotic force pushes water into the polymer network whereas dispersing force exerted by the polymer chains resist it. Increase in cross link density enhances the dispersing force. So with the increased cross linked density, a limited scope is available for free water to enter into the vacant spaces of cross linking network.

Fig. 1 ( $a$ and $b$ ) shows the effect of radiation dose on swelling ratio of $\mathrm{AAm}$ and $\mathrm{AAm} / \mathrm{CMC}$ blend hydrogels. It is found that swelling ratio of hydrogel decreases with increased radiation dose ${ }^{11}$. This result indicates that increased the radiation dose increases cross-linked density of hydrogels. Swelling of hydrogel is fast up to $24 \mathrm{~h}$ standing time in urea solution and then it becomes slow. The maximum value of swelling is obtained at $48 \mathrm{~h}$ standing in urea solution.

Water absorption of AAm and AAm/CMC blend hydrogels in distilled water and urea solutions

The maximum value of swelling for hydrogel may be called equilibrium swelling. Fig. 2 shows the water absorption percent of AAm and AAm/CMC blend hydrogels in distilled water and urea solutions. The swelling of hydrogels (AAm hydrogel and AAm/CMC blend hydrogel) in urea is higher than that of hydrogel in distilled water. The swelling of AAm hydrogel is $\sim 8252 \%$ and $6359 \%$ in $0.01 \mathrm{M}$ urea solution and distilled water respectively and the swelling ratio of $\mathrm{AAm} / \mathrm{CMC}$ blend hydrogel is $15921 \%$ and $13837 \%$ in $0.01 \mathrm{M}$ urea solution and distilled water respectively. This result can be explained by the fact that urea $\left(\mathrm{H}_{2} \mathrm{~N}-\mathrm{CO}-\right.$ $\mathrm{NH}_{2}$ ) is an organic compound and in distilled water it easily dissolves by the formation of hydrogen bond with water molecule. Because of the electro-negativity of nitrogen atom, hydrogen bonding is possible at both $\mathrm{N}-\mathrm{H}$ sites in the urea molecule ${ }^{12}$.

There is also a respectable polarity at the $\mathrm{C}=\mathrm{O}$ group and additional interactions can occur with hydrogel. Beside this, the urea solutions act as a weak base, which interacts with the carboxylic group of CMC. In our earlier study ${ }^{\mathbf{1 3}}$, it is found that swelling ratio of $\mathrm{AAm} / \mathrm{CMC}$ blend hydogel in inorganic salt sodium chloride $(\mathrm{NaCl})$ is lower than that of hydrogel in water.

\section{Effect of CMC on Swelling of AAm hydrogels}

From Fig. 3, it is also found that swelling ratio of $\mathrm{AAm} / \mathrm{CMC}$ blend hydrogel is higher than that of AAm hydrogel at similar radiation dose. AAm hydrogel in $0.01 \mathrm{M}$ urea solution, the equilibrium swelling value at $15 \mathrm{kGy}$ $8252 \%$. Whereas, AAm $/ 7 \% \mathrm{CMC}$ hydrogel in $0.01 \mathrm{M}$ urea solution, the equilibrium swelling values at $15 \mathrm{kGy}$ is 15921 $\%$.

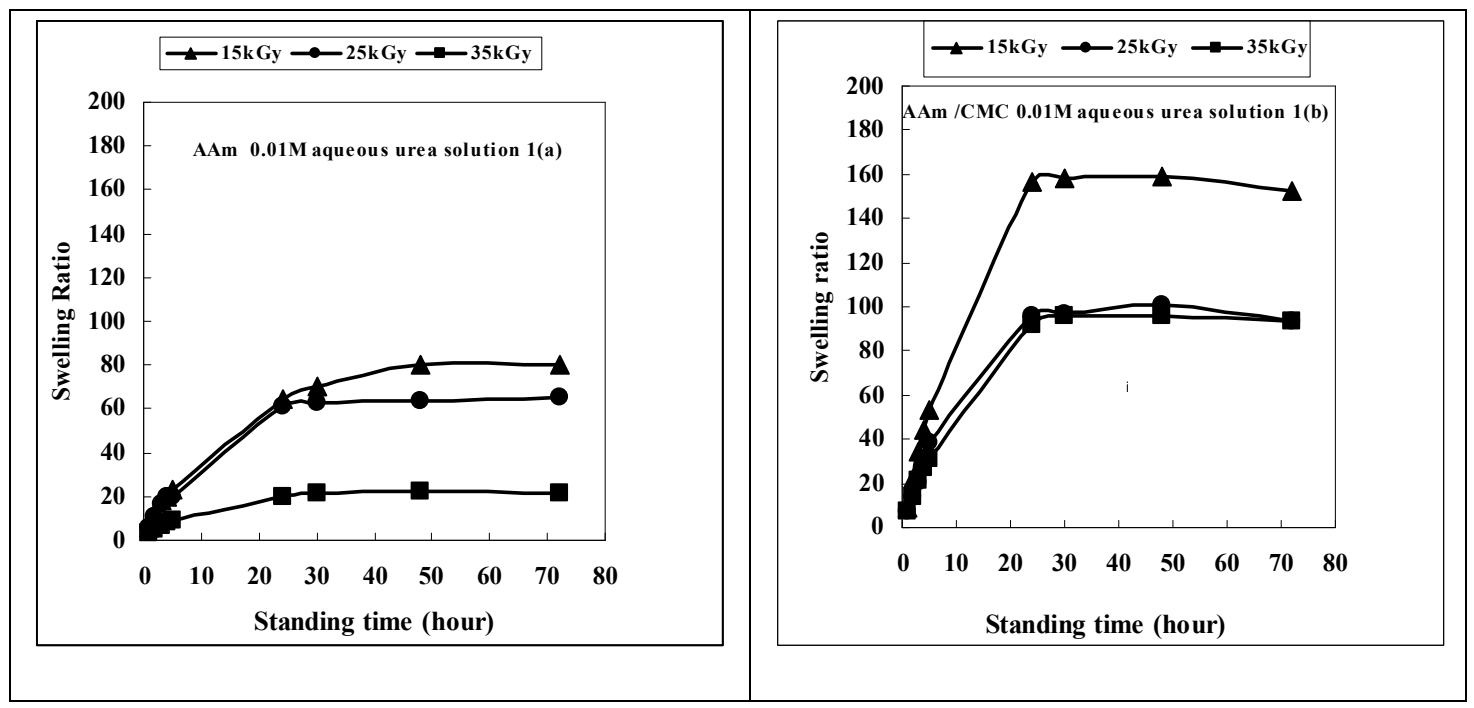

Fig. 1. Swelling Ratio of AAm and AAm/CMC blend hydrogels in urea solutions at different radiation doses. 
Fig. 2 .

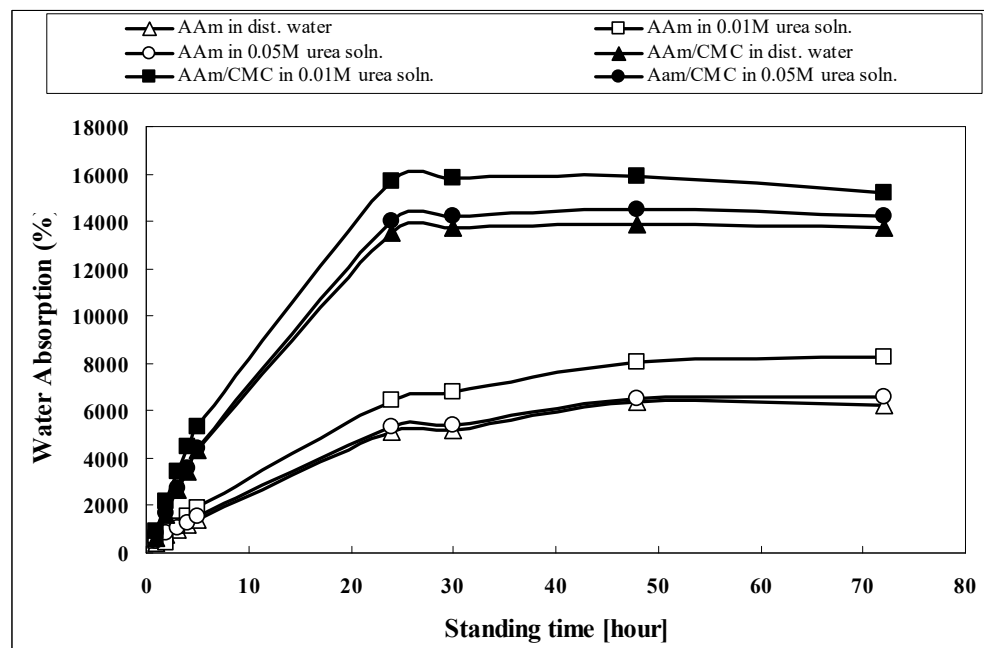

Figure 2: Water absorption of AAm and AAm/CMC blend hydrogels in distilled water and urea solution.

The cause of this is probably due to addition of CMC to AAm feed solution increases number of hydrophilic group in the hydrogel due to the presence of carboxyl group in the $\mathrm{CMC}$ molecules. On the other hand the more hydrophilic groups in the aqueous urea solutions get the more the swelling of the poly (AAm-co-CMC)hydrogels. In the presence of urea solution, swelling of poly (AAm-coCMC)hydrogels can easily follow the change of the hydrogen-bonded structure of water and polymer-solvent interaction $^{\mathbf{1 2}}$.

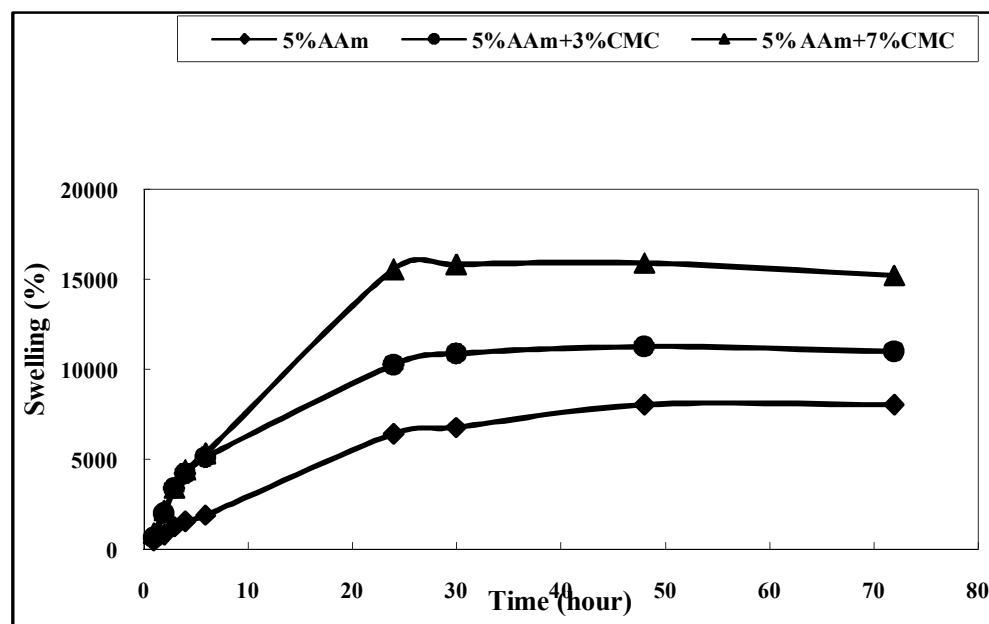

Fig. 3. Swelling of poly(AAm/CMC ) blend hydrogels in $0.01 \mathrm{M}$ urea solutions at $15 \mathrm{kGy}$.

\section{Conclusion}

It is clearly seen that the highest swelling percentages are observed in the AAm/CMC hydrogel system at the lower concentration $(0.01 \mathrm{M})$ of urea aqueous solution and swelling value increases with increasing the concentration of CMC. AAm hydrogel in $0.01 \mathrm{M}$ urea solution, the equilibrium swelling values of 15 and $35 \mathrm{kGy}$ are 8252 and $2255 \%$, respectively. Whereas, AAm $/ 7 \%$ CMC hydrogel in $0.01 \mathrm{M}$ urea solution, the equilibrium swelling values of 15 and $35 \mathrm{kGy}$ are 15921 and $9606 \%$, respectively. It has also observed that the values of the equilibrium swelling percent
$\left(\mathrm{S}_{\mathrm{eq}} \%\right)$ of swollen in urea solutions poly (AAm-co-CMC) blend hydrogels are higher than the hydrogels swollen in water. From the data presented in this paper, it can be concluded that poly (AAm-co-CMC) blend hydrogels are appropriate matrix for agricultural purpose as a soil conditioner.

\section{References}

1. Peppas, N. A. and A. G. Mikos, 1986. Preparation methods and structure of hydrogels. Hydrogels in medicine and pharmacy. Fundumentals, 1, CRC Press, Florida, 
2. Kim, S. W., Y. H. Bae and T. Okano, 1992. Hydrogels: swelling, drug loading, and release. Pharm. Res., 9, 283-290.

3. Byrne, M. E., K. Park and N. A. Peppas, 2002. Molecular imprinting within hydrogels. Adv. Drug Deliv. Rev., 54, 149-161.

4. Gupta, P., K. Vermani and S. Garg, 2002. Hydrogels: from controlled release to $\mathrm{pH}$-responsive drug delivery. Drug Discov. Today. 7, 569-579.

5. Karadag, E., O. B. Uzum, D. Saraydin and O. Guven, 2005. Swelling characterization of gamma-radiation induced CAMA hydrogels in urea solutions. Mater. Des. 26, 265-270.

6. Gargallo, L. and D. Radic, 1983. Interaction of polyvinylpyrrolidone with small cosolutes in aqueous and non aqueous media. Polymer. 24, 91-94.

7. Klotz, M. and K. Shikama, 1968. Nature of urea effect on anion binding by macromolecules. Arch. Biochem. Biophy. 123, 551-557.

8. Dafader, N. C., M. E. Haque and F. Akhtar, 2005. Effect of kappa-carrageenan on the properties of poly (vinyl pyrrolidone) hydrogel prepared by the application of radiation. Polym. Plast. Technol. Eng. 44, 1339-1346.

9. Dafader, N. C., A. Tahmina, M. E. Haque, S. P. Swapna, S. Islam and D. Huq, 2012. Effect of acrylic acid on the properties of polyvinylpyrrolidone hydrogel prepared by the application of gamma radiation, Afr. J. Biotech. 11, 1304913057.
10. Hashim, K., A. S. Halim, M. T. M. Nor, K. Z. M. Dahlan and F. Yoshii, 2002. PVA- Sago starch hydrogel and preliminary clinical animal study of the hydrogel. Proceeding of the Takasaki Symposium on Radiation Application of Natural Polymers in Asia, JAERI-Conf, Takasaki, Japan, No. 5, 19-31.

11. Zaki, A., 2007. Grafting of poly(vinyl pyrrolidone) with citric acid using gamma irradiation, Nucl. Instru. and Methods in Phy. Res., 265 (B), 179-182.

12. Karadag, E., O. Bars, D. Saraydin and O. Guven., 2005. Dynamic swelling behavior of $\gamma$-radiation induced polyelectrolyte poly (AAm-co-CA) hydrogels in urea solutions, Inter. J. of Pharm.ceutics, 301, 102-111.

13. Sultana, S., M. R. Islam, N. C. Dafader, M. E. Haque, 2012. Preparation of carboxymethyl cellulose/acrylamide copolymer hydrogel using gamma radiation and investigation of its swelling behavior. J. Bang. Chem. Soc. 25, 132-138. 
Fig. 2.

Fig. 2.

Fig. 2. 